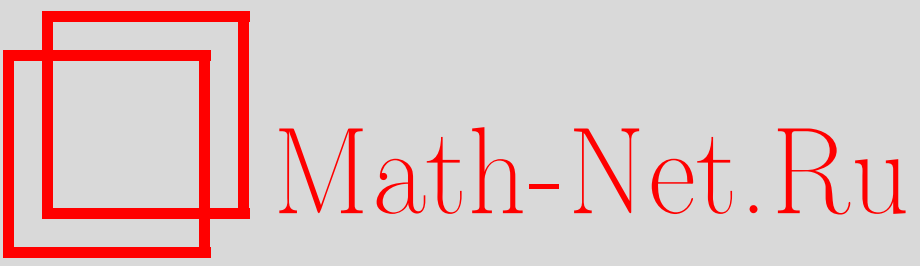

Р. Ф. Вернер, А. С. Холево, М. Е. Широков, О понятии сцепленности в гильбертовых пространствах, УМH, 2005, том 60, выпуск 2, 153-154

DOI: https://doi.org/10.4213/rm1411

Использование Общероссийского математического портала Math-Net.Ru подразумевает, что вы прочитали и согласны с пользовательским соглашением

http://www.mathnet.ru/rus/agreement

Параметры загрузки:

IP: 3.91 .87 .62

26 апреля 2023 г., 16:44:14 


\title{
О ПОНЯТИИ СЦЕПЛЕННОСТИ В ГИЛЬБЕРТОВЫХ ПРОСТРАНСТВАХ
}

\author{
Р. Ф. ВеРнеР, А. С. Холево, М.Е. ШИРОков
}

Оператор плотности (состояние) в тензорном произведении $\mathscr{H} \otimes \mathscr{K}$ гильбертовых пространств называется разделим ьцм, если он принадлежит вьпуклому замьканию подмножества состояний-произведений. Неразделимые состояния назьваются сцепленными (entangled). Эти понятия имеют важное значение в квантовой теории информации, однако они подробно изучались только в конечномерном случае [1]. В этом сообщении дается общее интегральное представление для разделимых состояний и впервые построен пример разделимого состояния, которое не является счетно разложимым. Мы также доказьваем структурную теорему для квантовых каналов, разрушающих сцепленность (entanglement-breaking), которая обобщает конечномерный результат из [2]. В конечномерном случае такие каналы могут быть охарактеризованы как имеющие представление Стайнспринга-Крауса (3) с операторами $V_{j}$ ранга 1 . Пример не счетно разложимого состояния показьвает существование бесконечномерных каналов, разрушающих сцепленность, которые не имеют такого представления.

В далшнейшем $\mathscr{H}, \mathscr{K}, \ldots$ - сепарабельные гильбертовы пространства, $\mathfrak{T}(\mathscr{H})$ - банахово пространство ядерных операторов и $\mathfrak{S}(\mathscr{H})$ - выпуклое множество всех операторов плотности в $\mathscr{H}$. Для краткости мы называем их состояниями, подразумевая, что оператор плотности $\rho$ однозначно задает нормальное состояние на алгебре $\mathfrak{B}(\mathscr{H})$ всех ограниченных операторов в $\mathscr{H}$. Множество $\mathfrak{S}(\mathscr{H})$ с расстоянием, определяемьм следовой нормой, является полным сепарабельным метрическим пространством. Если $\pi$ - борелевская вероятностная мера на $\mathfrak{S}(\mathscr{H})$, то интеграл Бохнера

$$
\bar{\rho}(\pi)=\int_{\mathfrak{S}(\mathscr{H})} \sigma \pi(d \sigma)
$$

определяет состояние, назьваемое барицентром меры $\pi$.

Следующая лемма, которая усиливает утверждение о разложении Шоке для случая замкнутых вьпуклых подмножеств $\mathfrak{S}(\mathscr{H})$, доказьвается с использованием критерия компактности

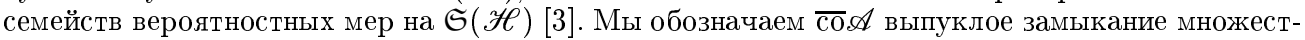
ва $\mathscr{A}[4]$.

Лемма. Пусть $\mathscr{A}$ - замкнутое подмножество $\mathfrak{S}(\mathscr{H})$. Тогда со $\mathscr{A}$ совпадает с множеством барицентров всех борелевских вероятностных мер с носителем $\mathscr{A}$.

ОпредЕлЕниЕ 1. Состояние в $\mathscr{H} \otimes \mathscr{K}$ называется разделимым, если оно принадлежит выпуклому замьканию подмножества всех состояний-произведений $\rho \otimes \sigma$, где $\rho \in \mathfrak{S}(\mathscr{H}), \sigma \in \mathfrak{S}(\mathscr{K})$. Состояние назьвается сцепленным, если оно не разделимо.

В данном определении множество всех состояний-произведений можно заменить на подмножество всех чистых состояний-произведений (крайних точек множества $\mathfrak{S}(\mathscr{H} \otimes \mathscr{K})$ ). Подмножество $\mathfrak{P}(\mathscr{H} \otimes \mathscr{K})$ чистых состояний замкнуто в топологии следовой нормы. Поэтому из леммы следует, что состояние $\rho$ является разделимым тогда и только тогда, когда существует борелевская вероятностная мера $\mu$ на $\mathfrak{P}(\mathscr{H}) \times \mathfrak{P}(\mathscr{K})$ такая, что

$$
\rho=\int_{\mathfrak{P}(\mathscr{H})} \int_{\mathfrak{P}(\mathscr{K})}|\varphi\rangle\langle\varphi|\otimes| \psi\rangle\langle\psi| \mu(d \varphi d \psi) .
$$

В конечномерном случае в силу теоремы Каратеодори данное представление сводится к известному определению разделимого состояния как конечной выпуклой комбинации чистых состояний-произведений [1]. В общем случае назовем состояние счетно разложимым, если для него существует представление (2) с чисто атомической мерой $\mu$.

Канал - это линейное сохраняющее след отображение из $\mathfrak{T}(\mathscr{H})$ в $\mathfrak{T}\left(\mathscr{H}^{\prime}\right)$ такое, что двойственное отображение $\Phi^{*}: \mathfrak{B}\left(\mathscr{H}^{\prime}\right) \mapsto \mathfrak{B}(\mathscr{H})$ (которое существует, посколку $\Phi$ ограничено) является вполне положительным. Для любого канала существует (неединственное) представление Стайнспринга-Крауса

$$
\Phi(\rho)=\sum_{j} V_{j} \rho V_{j}^{*}
$$

в котором $V_{j}$ - ограниченные операторы из $\mathscr{H}$ в $\mathscr{H}^{\prime}$ такие, что $\sum_{j} V_{j}^{*} V_{j}=I$.

Работа выполнена при поддержке фонда А. фон Гумболшдта и гранта НШ 1758.2003.1. 
ОПРеДЕЛЕниЕ 2. Канал $\Phi$ называется каналом, разрушающим сцепленность, если для любого гильбертова пространства $\mathscr{K}$ и любого состояния $\omega \in \mathfrak{S}(\mathscr{H} \otimes \mathscr{K})$ состояние $\left(\Phi \otimes \operatorname{Id}_{\mathscr{K}}\right)(\omega)$, где $\operatorname{Id}_{\mathscr{K}}$ - тождественный канал в $\mathscr{K}$, является разделимым.

Теорема 1. Канал Ф является каналом, разрушающим сцепленность, тогда и только тогда, когда существует полное сепарабельное метрическое пространство $\mathscr{X}$, борелевская $\mathfrak{S}\left(\mathscr{H}^{\prime}\right)$-значная функиия $x \mapsto \rho^{\prime}(x)$ и разложсение единицы (положительная операторно-значная мера) $M(d x)$ на $\mathscr{X}$ такие, что

$$
\Phi(\rho)=\int_{\mathscr{X}} \rho^{\prime}(x) \mu_{\rho}(d x)
$$

где $\mu_{\rho}(B)=\operatorname{Tr} \rho M(B)$ для любого борелевского множества $B \subseteq \mathscr{X}$.

Доказательство использует обобщение известного соответствия между вполне положительными отображениями и состояниями в $\mathscr{H}^{\prime} \otimes \mathscr{K}[5]$.

Если существует представление (4) с чисто атомической мерой $M(d x)$, то канал $\Phi$ называется счетно разложиимым. Нетрудно убедиться, что канал является счетно разложимым тогда и только тогда, когда для него существует представление (3) с операторами $V_{j}$ ранга 1. $\mathrm{C}$ другой стороны, канал является счетно разложимым тогда и только тогда, когда состояния в $\left(\Phi \otimes \operatorname{Id}_{\mathscr{K}}\right)(\mathfrak{S}(\mathscr{H} \otimes \mathscr{K}))$ счетно разложимы. Это сводит вопрос о существовании канала, разрушающего сцепленность, но не имеющего представления (3) с операторами ранга 1, к вопросу о существовании разделимого состояния, которое не является счетно разложимым. Ниже приведена конструкция такого состояния.

Рассмотрим одномерньй тор $\mathbb{T}$, параметризованньй как интервал $[0,2 \pi)$ со сложением $\bmod 2 \pi$. Пусть $\mathscr{H}=L^{2}(\mathbb{T})$ с нормированной мерой Лебега $\frac{d x}{2 \pi}$. Рассмотрим унитарное представление $x \rightarrow V_{x}$ группы $\mathbb{T}$, где $\left(V_{u} \psi\right)(x)=\psi(x-u)$.

Теорема 2. Для любых векторов состояний $\left|\varphi_{j}\right\rangle \in \mathscr{H}_{j} \simeq L^{2}(\mathbb{T}), j=1,2$, с отличнылми от нуля коэффициентами Фурье разделимое состояние

$$
\rho_{12}=\int_{0}^{2 \pi} V_{x}^{(1)}\left|\varphi_{1}\right\rangle\left\langle\varphi_{1}\left|V_{x}^{(1) *} \otimes V_{x}^{(2)}\right| \varphi_{2}\right\rangle\left\langle\varphi_{2}\right| V_{x}^{(2) *} \frac{d x}{2 \pi}
$$

в $\mathfrak{S}\left(\mathscr{H}_{1} \otimes \mathscr{H}_{2}\right)$ не является счетно разложимым.

СледСтвиЕ 1. Существует канал, разрушающий сцепленность, не представимьй в виде (3) с операторами $V_{j}$ ранга 1.

Доказательство состоит в явном построении, опирающемся на пример теоремы 2 и соответствие между вполне положительными отображениями и состояниями в тензорном произведении пространств. Имеется гипотеза, что не счетно разложимые состояния образуют плотное подмножество во множестве всех разделимых состояний. В пользу этого говорит следующий результат.

СледСтвиЕ 2. В любой окрестности произвольного чистого состояния-произведения найдутся разделимые состояния, которье не счетно разложимы.

\section{СПИСОК ЛИТЕРАТУРЫ}

[1] R. F. Werner. Quantum Information Theory - an Invitation // Springer Tracts Modern Phys.. V. 173, 2001; e-print quant-ph/0101061. [2] M. Horodecki, P. W. Shor, M. B. Ruskai // Rev. Math. Phys. 2003. V. 15. P. 629-641; e-print quant-ph/0302031. [3] A. C. Холево, M. Е. Широков // Теория вероятн. и ее примен. 2005 (to appear); e-print quant-ph/0408176. Т. 50. № 1. [4] А. Д. Иоффе, В. М. Тихомиров. Теория экстремальных задач. М.: Наука, 1974. [5] M.-D. Choi // Linear Algebra Appl. 1975. V. 10. P. 285-290.

Institute of Mathematical Physics, TUB,

Braunschweig, Germany;

Представлено А. В. Булинским

Математический институт им. В. А. Стеклова РАН

Принято редколлегией 15.03.2005

E-mail: holevo@mi.ras.ru, msh@mi.ras.ru 